\title{
Management of solitary fibrous tumours of the pleura: a systematic review and meta-analysis
}

\author{
Rachel M. Mercer ${ }^{1,2}$, Charlotte Wigston ${ }^{1}$, Radhika Banka ${ }^{1}$, Giuseppe Cardillo ${ }^{3}$, \\ Rachel Benamore ${ }^{1}$, Andrew G. Nicholson ${ }^{4}$, Rachelle Asciak ${ }^{1,2}$, Maged Hassan (1) ${ }^{1,2,5}$, \\ Robert J. Hallifax ${ }^{1,2}$, Louise Wing ${ }^{1}$, Eihab O. Bedawi ${ }^{1,2}$, Nick A. Maskell ${ }^{6}$, \\ Elinor K. Harriss ${ }^{7}$, Robert F. Miller ${ }^{8}$ and Najib M. Rahman ${ }^{1,2,9}$
}

Affiliations: ${ }^{1}$ University of Oxford Respiratory Trials Unit, Churchill Hospital, Oxford, UK. ${ }^{2}$ Oxford Centre for Respiratory Medicine, Oxford University Hospitals NHS Trust, Oxford, UK. ${ }^{3}$ San Camillo Forlanini Hospital, Rome, Italy. ${ }^{4}$ Dept of Histopathology, Royal Brompton and Harefield NHS Foundation Trust, and National Heart and Lung Institute, Imperial College, London, UK. ${ }^{5}$ Chest Diseases Department, Faculty of Medicine, Alexandria University, Alexandria, Egypt. ${ }^{6}$ Academic Respiratory Unit, Bristol Medical School, Southmead Hospital, University of Bristol, Bristol, UK. ${ }^{7}$ Bodleian Health Care Libraries, University of Oxford, Oxford, UK. ${ }^{8}$ Institute for Global Health, University College London, London, UK. ${ }^{9}$ NIHR Oxford Biomedical Research Centre, University of Oxford, Oxford, UK.

Correspondence: Rachel M. Mercer, University of Oxford Respiratory Trials Unit, Churchill Hospital, Oxford, OX3 7LE, UK. E-mail: Rachel.mercer@anhs.net

\section{ABSTRACT}

Background: Solitary fibrous tumours of the pleura (SFTP), or pleural fibromas, are rare tumours that generally, but not universally, follow a benign course. Surgical resection is the standard treatment, but there are no evidence-based guidelines regarding the management of these tumours.

Methods: Five databases were searched from inception to April 1, 2019 for studies reporting on SFTP management.

Results: Twenty-seven studies met the inclusion criteria (1542 patients, all non-comparative case series); 98\% of these patients underwent resection and all SFTP included were pathologically diagnosed. 394 out of 1299 cases $(30.5 \%, 95 \%$ CI $27.8-32.8 \%)$ were malignant with recurrence rates of between $0 \%$ and $42.9 \%$. A pleural effusion was always associated with a negative outcome, but no other features were consistently reported to have negative associations. Preoperative biopsies incorrectly reported malignant histology in two studies. Over $25 \%$ of cases of recurrence occurred when a complete (R0) resection had been achieved. The first recurrence occurred $>5$ years after the initial resection in at least $23 \%$ of cases.

Conclusions: There is strong evidence to support long-term surveillance after surgical resection of SFTP, even where a complete (R0) resection has been achieved; however, there is no clear evidence to inform clinicians regarding the selection of patients who should undergo resection. The rates of malignant SFTP and SFTP recurrence are higher than previously reported. Only those that were pathologically diagnosed or resected were included, which may bias the data towards more aggressive tumours. Data collection on radiologically diagnosed SFTP is required to draw conclusions regarding the timing and need for intervention.

@ERSpublications

Long-term surveillance should be undertaken after a resection of solitary fibrous tumours of the pleura but further work is needed to determine which patients are likely to follow a malignant clinical course to decide timing and necessity of a resection https://bit.ly/2U10SaA

Cite this article as: Mercer RM, Wigston C, Banka R, et al. Management of solitary fibrous tumours of the pleura: a systematic review and meta-analysis. ERJ Open Res 2020; 6: 00055-2020 [https:// doi.org/10.1183/23120541.00055-2020].

This article has supplementary material available from openres.ersjournals.com

Received: 4 Feb 2020 | Accepted after revision: 26 May 2020

Copyright $\odot$ ERS 2020. This article is open access and distributed under the terms of the Creative Commons Attribution Non-Commercial Licence 4.0. 


\section{Introduction}

Solitary fibrous tumours are rare, slow-growing tumours arising from mesenchymal cells. They were first described in the pleura but can arise at multiple extra-thoracic sites. Solitary fibrous tumours of the pleura (SFTP) commonly arise from the visceral rather than the parietal pleura and are often larger than $10 \mathrm{~cm}$ [1]. Many different terms have been used to describe these tumours such as localised mesothelioma, hemangiopericytoma, localised benign fibroma and localised fibrous tumour, but SFTP is now the accepted terminology in the most recent World Health Organization (WHO) classification [2].

Radiologically, SFTP are often homogeneous, well-defined, noninvasive tumours abutting the chest wall, making an obtuse angle at the pleural surface [3]. There is a wide differential diagnosis of imaging including both primary and secondary mesenchymal soft tissue tumours, localised mesothelioma [4] and peripheral lung neoplasms [5].

SFTP account for less than $5 \%$ of pleural-based tumours, and their clinical course can be unpredictable. ENGLAND et al. [1] proposed criteria to differentiate tumours with malignant or benign features, but these criteria do not always predict a malignant clinical course [6]. Treatment typically involves surgical resection, which is usually curative. A minority of patients also undergo chemotherapy or radiotherapy.

There is no consensus guideline on management of SFTP, and the evidence base for treatment is limited, leading to large variations in care. This systematic review aims to appraise the current evidence base for the management of SFTP to provide up-to-date information for clinicians in the management of this disease entity.

\section{Methods}

This systematic review adhered to the PRISMA reporting standards and has been registered on the PROSPERO database (ID CRD42018091527).

The specific questions addressed were:

- Which patients need intervention for their SFTP?

- What follow-up should be undertaken for patients with SFTP who have had conservative management?

- What follow-up should be undertaken for patients who have had resection of SFTP?

- Is there any role for chemotherapy or radiotherapy in patients with SFTP?

\section{Literature search}

PubMed, Ovid Embase, the Cochrane Central Register of Controlled Trials, Scopus and Web of Science Core Collection were searched from inception to April 1, 2019. Search terms combined synonyms, acronyms and the thesaurus terms for "pleural fibroma", SFTP*, Solitary Fibrous Tumour and Pleural/, as well as using adjacency commands to search for the phrase "solitary fibrous tum*" appearing within three words of "pleura*". The results were limited to English language publications only. Full search terms are documented in appendix 1 .

\section{Eligibility criteria}

All studies that detailed medical and surgical management and clinical outcomes of patients with SFTP were included. Due to lack of randomised control trials, case series were included. Although rare, over 1000 cases have been reported in the literature with multiple case series of over 100 patients: any article reporting fewer than 20 cases of SFTP was excluded. Abstracts only were first reviewed by the authors, and those clearly out of scope excluded.

Duplicates were removed along with non-English papers, those with only a title and no abstract, and articles without available full texts. Exclusion criteria included non-primary studies (review articles and opinion pieces), articles without clinical outcome data specific to SFTP and papers where the primary investigative question was related to histological tests without clearly relevant clinical data. Full text papers were obtained and were excluded for any of the above criteria. Where data from the same hospital were represented in multiple publications, the paper which provided the largest amount of relevant data was included.

\section{Data extraction}

Two reviewers screened abstracts using the inclusion criteria, then assessed the full text articles against the inclusion and exclusion criteria and extracted data via a predesigned database. A third reviewer resolved any disagreements.

Data collected included:

- Lead author

- Year published 
- Years covered

- Geographical area

- Retrospective/prospective

- Number of SFTP cases

- Pleural alone or all SFT

- Rates and definition of malignancy

- Resection rates

- Operative morbidity and mortality

- Recurrence rate, local or distant, and time to recurrence

- Survival

- Chemotherapy or radiotherapy treatment

- Follow-up duration

Data synthesis was planned as either a meta-analysis or narrative review, depending on the consistency and quality of data discovered. Statistics were carried out using the open-source software "Open meta analyst" (www.cebm.brown.edu/openmeta).

\section{Definitions}

A malignant SFTP was defined using histological criteria including: $>4$ mitotic figures per 10 high-power fields, pleomorphism, hypercellularity, haemorrhage and/or necrosis [1] (details in appendix 2), rather than invasion into surrounding structures or metastases, which were reported separately. Features suggesting a malignant clinical course were reported including recurrence and metastatic disease.

\section{Results}

\section{Extracted studies}

A total of 3958 publications were assessed after deduplication. Of these, 2848 were not related to the study questions, 610 did not have appropriate primary data (reviews, case series $<20$ pleural cases, primary histological study), and 440 had only a title and no abstract. Of the 60 remaining abstracts, seven did not have an associated full text, and in one case the full text could not be obtained. Ten papers were excluded as they either assessed the same cohorts of patients or included patients from other studies in the review (appendix 3). One paper focussed on intrapulmonary (rather than pleural) fibromas, and eight others had $<20$ pleural cases on more detailed inspection. In three papers, it was not possible to extract pleural-specific data, and three did not have sufficient data regarding patient outcomes. This resulted in 27 papers for data extraction [1,5-30], which included a total of 1542 SFTP (figure 1).

\section{Study characteristics}

Among the 27 papers, there were no randomised or case-control comparative studies, with all papers being case series only. Only 2 out of 27 (7\%) papers reported prospectively collected data, accounting for 126 cases $[15,29]$.

Due to the heterogeneity of data, meta-analysis was only considered appropriate in one instance; therefore, narrative summaries are provided. No paper addressed clinical or radiological follow-up for conservatively managed (non-resected, radiologically diagnosed) SFTP.

\section{Baseline information}

The number of participants in each paper ranged from 21(22, 25) to 223(1) over a time period from 1977 to 2015. Geographical locations included Asia, Europe and North America. Median and mean ages ranged from 41-66 years (appendix 4).

In all 27 papers, patients had a histological diagnosis of SFTP; 1454 out of 1485 (98\%) patients underwent resection of their SFTP. Median duration of follow-up ranged from 27 to 146 months (appendix 5).

\section{Rates of malignancy and recurrence}

A definition of malignancy was provided in 21 out of 27 studies, all of which included histological criteria only. The rates of malignant SFTP ranged from $10.3 \%$ to $57 \%[8,16]$, but there was some heterogeneity in the definition of malignancy among papers (appendix 2). A meta-analysis of studies that used the criteria proposed by England et al.(appendix 2) showed a rate of malignancy of 307 out of $961(29.0 \%, 95 \%$ CI $20.6 \%-37.5 \%$ ) cases but with an $\mathrm{I}^{2}$ value of $89.05 \%$, suggesting significant heterogeneity (figure 2 ). An overall summary of all studies using all definitions demonstrated 394 out of 1299 (30.5\%, 95\% CI $27.8 \%-32.8 \%$ ) cases of SFTP were diagnosed as malignant.

The reported recurrence rate was between $0 \%$ and $42.9 \%[9,12]$, but how this was calculated varied among papers. A conservative estimate of recurrence uses the original number of cases of SFTP included 


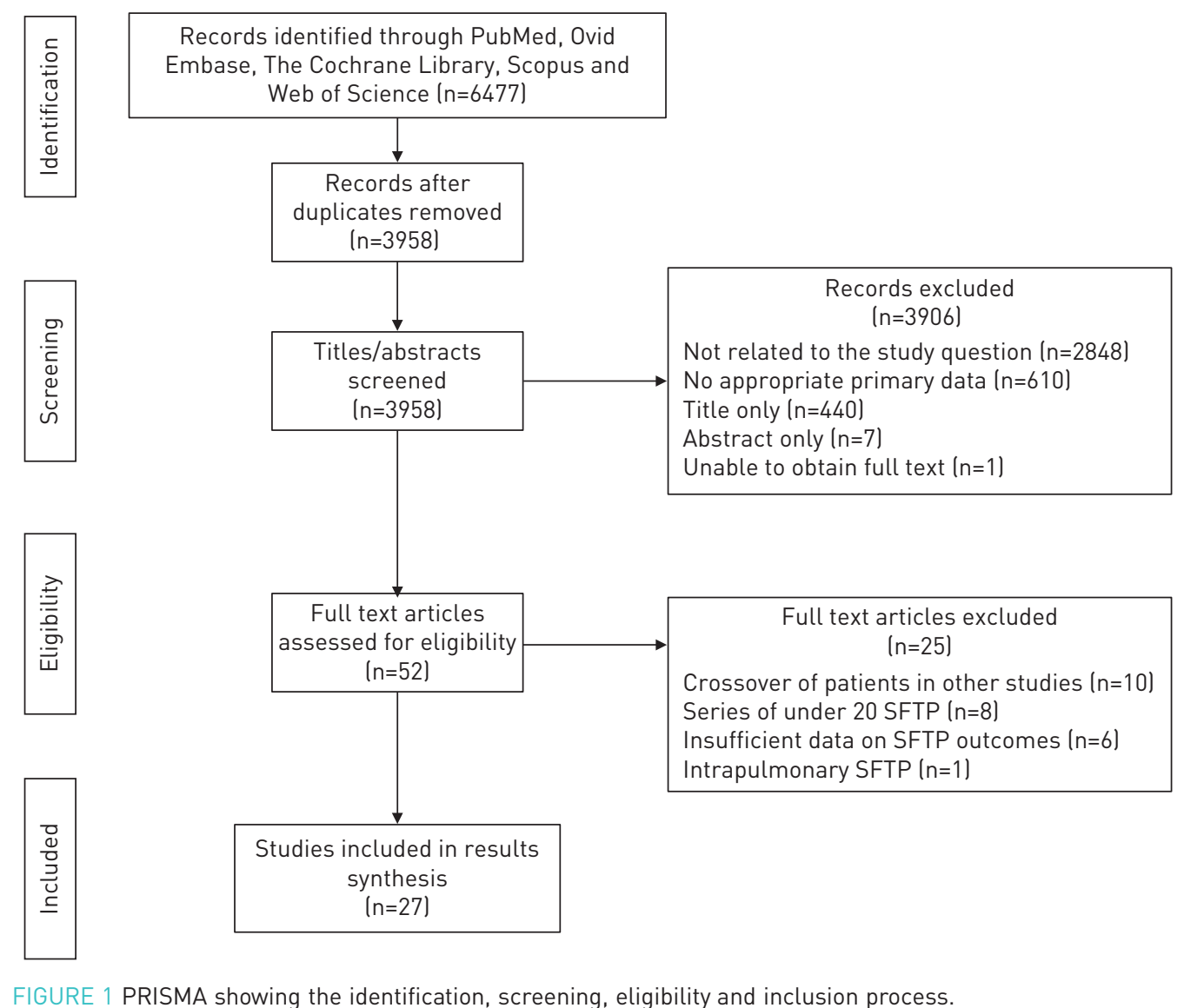

in the studies as the denominator and cases of recurrence as the numerator, 154 out of 1330 (11.6\%, 95\% CI 9.9-13.3\%). When the recurrence rates with the denominator determined by the authors of the paper were combined, 154 out of 1179 (13.1\%, 95\% CI 11.1\%-15.0\%) patients experienced tumour recurrence. When analysis included only papers where $100 \%$ of SFTP were resected and over $95 \%$ of cases had "complete" follow-up, 50 out of 635 (7.9\%, 95\% CI 5.8\%-10.0\%) recurred. At least 18 out of $652(2.8 \%$, 95\% CI 1.5\%-4.0\%) patients with histologically "benign” SFTP experienced recurrence.

Studies
Boddeart
Bylicki
Cardillo
DeVito
England
Guo
Helage
Lahon
Liu
McGuire
Santos
Tan
Yeom

Overall $\left(\left.\right|^{2}=89.05 \%, p<0.001\right)$
Estimate $(95 \% \mathrm{CI})$

$0.354(0.249-0.460)$

$0.574(0.456-0.691)$

$0.136(0.072-0.200)$

$0.464(0.280-0.649)$

$0.368(0.304-0.431)$

$0.103(0.007-0.198)$

$0.411(0.282-0.540)$

$0.427(0.349-0.504)$

$0.143(0.000-0.293)$

$0.348(0.153-0.542)$

$0.206(0.070-0.342)$

$0.146(0.070-0.223)$

$0.146(0.038-0.255)$

$0.290(0.206-0.375)$

Malig/overall

$28 / 79$
$39 / 68$
$15 / 110$
$13 / 28$
$82 / 223$
$4 / 39$
$23 / 56$
$67 / 157$
$3 / 21$
$8 / 23$
$7 / 34$
$12 / 82$
$6 / 41$

$307 / 961$

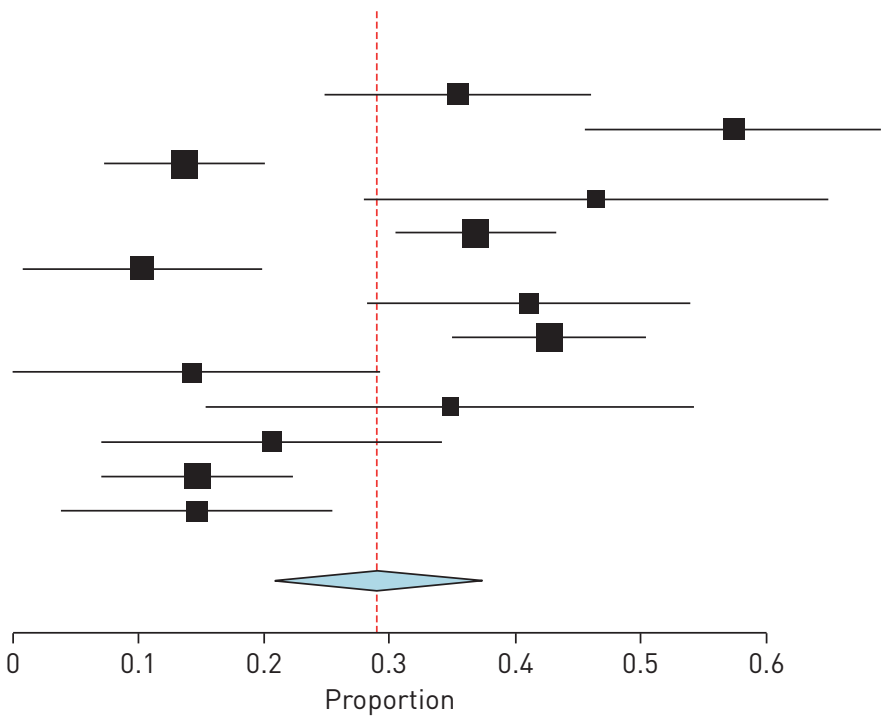

FIGURE 2 Meta-analysis of malignant rates using criteria from England et al. [1]. 
Operative mortality

The 30 -day operative mortality ranged from $0 \%$ to $3.6 \%$ [18]. Morbidity rates were between $0 \%$ and $16.7 \%$, but there was no standardised reporting across the studies (appendix 6).

\section{Predicting the need for intervention}

No studies adequately addressed the true rate of patients undergoing intervention. No study reported an appropriate denominator, i.e. those who were diagnosed radiologically. The published literature provided insufficient data regarding outcomes for patients who were conservatively managed to use as a comparator ( $<2 \%$ of the patients in the review, many of whom had proven metastatic disease).

\section{Preoperative biopsies}

Sixteen studies reported on preoperative transthoracic biopsies, which included 216 patients (appendix 7). The diagnostic yield from fine-needle aspiration was between $8 \%$ (17) and 45\% (7), and from core needle biopsies between $27 \%$ (30) and 100\% (17). One study reported two of three SFTP classified as malignant on biopsy were benign when resected [23], but another found lower mitotic counts in biopsy specimens compared to subsequently resected tumours [11].

There were no reported instances in this review of tumour seeding after transthoracic biopsy, although it was noted by CARDILLO et al. [6] that fine-needle aspiration had been performed in one of the eight cases where recurrence occurred.

\section{Follow-up after surgical resection}

Predictors of outcomes

Larger tumour size was found to be associated with malignant histology in seven papers (710 cases), four of which used a cut-off of $10 \mathrm{~cm}$. There was no apparent association in a further three papers. A greater size at diagnosis was only associated with a poor clinical outcome in two papers and a further three papers did not find an association. Age and sex had no significant association with a malignant outcome. Six papers reported an association between malignant histology and poor clinical outcomes. Two further papers did not show an association. Only three studies found significant negative associations between parietal origin or sessile attachment, whereas nine found no significant association. The presence of a pleural effusion was found to be associated with malignant histology and poorer outcomes in all papers where this was assessed. Pleural fluid cytology was positive in only one reported case. Further details are shown in table 1.

Incomplete resection [8], multifocal disease and extensive surgical procedures [21], fibrous adherences [7], fluorodeoxyglucose-positron emission tomography $\left({ }^{18}\right.$ FDG PET) avidity [5], lobulated borders [17] and inverted tumours were associated with negative outcomes in single studies. A further study reported multiple computed tomography (CT) features predictive of malignancy [19].

\section{Time to recurrence}

Time to recurrence data were available in 12 papers, which included 73 patients. Extrapolating from this statistical information, 35 out of $73(47.9 \%)$ cases demonstrated a first recurrence within 5 years, 17 out of $73(23.3 \%)$ cases had a first recurrence occurring after 5 years, and it was not possible to establish timings in the remaining $21(28.8 \%)$ cases. At least 39 cases demonstrated recurrence in populations where complete (R0) resection had been achieved in $100 \%$ of the cases $[6,14,16,19,23,25,26,29]$.

Where information was available, re-resection for patients with recurrence occurred in 50 out of 90 (65.6\%) cases, and 14 out of $23(60.9 \%)$ cases showed recurrence at least one further time after the repeat operation.

\section{Survival outcomes}

Overall, 5-year survival and disease-free survival in patients after resection ranged between $83 \%$ and $92.4 \%$ and $71 \%$ and $87.2 \%$ respectively (appendix 8 ).

\section{Chemotherapy and radiotherapy}

Only one study used chemotherapy or radiotherapy routinely in the treatment pathway [8]. Of the remaining cases, only 46 out of 1014 (4.5\%) underwent oncological treatments (appendix 9).

\section{Discussion}

This review demonstrates the wide heterogeneity of clinical management of, and outcomes for, patients with SFTP. 
TABLE 1 Statistically significant predictors of malignancy and outcomes

\begin{tabular}{|c|c|c|c|c|}
\hline & $\begin{array}{l}\text { Predictive of histological } \\
\text { malignancy }\end{array}$ & $\begin{array}{l}\text { No association with } \\
\text { histological malignancy }\end{array}$ & $\begin{array}{l}\text { Predictive of reduced } \\
\text { survival or recurrence }\end{array}$ & $\begin{array}{l}\text { No association } \\
\text { with poor } \\
\text { outcomes }\end{array}$ \\
\hline $\begin{array}{l}\text { Parietal rather than } \\
\text { visceral tumour } \\
\text { origin }\end{array}$ & England (223) & $\begin{array}{c}\text { McGuire (23), Cardillo (110), } \\
\text { Harrison-Phipps (84), } \\
\text { Boddeart (80), Lahon (157), } \\
\text { Tan (82) }\end{array}$ & Tapias - RFS (59) & $\begin{array}{l}\text { McGuire (23), } \\
\text { Cardillo (110), } \\
\text { Franzen (42) }\end{array}$ \\
\hline $\begin{array}{l}\text { Intra-tumoural } \\
\text { calcification }\end{array}$ & Gupta (28) & Helage (56) & & \\
\hline $\begin{array}{l}\text { Malignant features } \\
\text { on histology }\end{array}$ & $\mathrm{N} / \mathrm{A}$ & N/A & $\begin{array}{c}\text { Franzen - DFS (42), Lahon - } \\
\text { DFS (157), Boddeart - DFS } \\
\text { (80), Cardillo - DFS (110), } \\
\text { Harrison-Phipps - } \\
\text { recurrence and OS (84) }\end{array}$ & $\begin{array}{l}\text { McGuire (23), } \\
\text { Franzen (42), } \\
\text { Boddeart - OS (80) }\end{array}$ \\
\hline
\end{tabular}

Bold type: remains significant on multivariate analysis; roman type: multivariate analysis not undertaken; italic type: no longer significant on multivariate analysis. Numbers in parenthesis are the number of patients in the study. DFS: disease-free survival; RFS: recurrence-free survival; OS: overall survival.

Rates of malignancy

Combining information from all studies suggests that the rate of malignant histological features in SFTP is around $30 \%$.

\section{Predicting the need for intervention}

There is evidence to support the assumption that larger tumours are associated with histological malignancy, but not necessarily a malignant clinical course. A cut-off value of $10 \mathrm{~cm}$ was often used, but a more clinically relevant cut-off may be much lower, to try and establish whether there is a threshold at which tumours are unlikely to have a malignant clinical course. The presence of a pleural effusion was the only clinical feature that consistently had negative associations. Due to the heterogeneity of studies, significant predictors associated with clinical outcome could have been missed due to lack of analysable data and thus inability to perform a meta-analysis. It is possible that associations which were found to be non-significant in individual papers were due to small sample size.

Preoperative percutaneous biopsy has a low sensitivity, and the results may not correlate with surgical resection specimens, especially in the diagnosis of malignancy [11, 23]. Complication rates of the procedures were not reported. There is one recorded instance of tumour seeding after a CT-guided biopsy [31], but there was no evidence of this complication in the present review.

\section{Follow-up after surgical resection}

In the host centre (Oxford, UK), patients with SFTP are currently followed up for 5 years. However, of those who develop recurrence, in at least $23 \%$ the first recurrence occurs $>5$ years after resection. Further information is needed regarding the peak time of recurrence to determine whether more intensive screening is required initially and to identify which patients are at risk of late recurrence.

\section{Recurrence}

The rate of recurrence was between $8 \%$ and $13 \%$, depending on whether all cases or only those with a successful resection were included. A cohort of 50 patients with malignant SFTP, 92\% of whom had a 
complete resection, demonstrated a recurrence rate of 30\% [32], which re-enforces the idea that malignant SFTP are likely to have a higher rate of relapse. Operative morbidity is low, and there is good 5-year survival after surgery in selected patients, but there is no evidence to describe outcomes should a patient not undergo surgery. A second recurrence was found in $61 \%$ of patients after re-resection had been undertaken, which may infer that the innate characteristic of the tumour determines outcome, rather than success of surgical resection.

Identifying which patients are at a higher risk of recurrence would be useful as it may be possible to tailor follow-up schedules or to consider additional oncological interventions in this group. A number of post-operative scoring systems have been proposed that could be of help in identifying these patients [11, $29,33,34$ ], but although validated [35], up to $20 \%$ of patients considered low risk experienced recurrence in one analysis [36].

\section{Limitations and bias}

The published data are non-randomised, non-comparative and mostly retrospective, resulting in a high likelihood of bias, although there are no validated "risk of bias" tools for formal assessment of descriptive case series.

In this review, due to lack of data it was not possible to address the questions of timing of intervention or conservative management of SFTP. Likewise, evidence-based conclusions could not be made regarding oncological treatment due to the small number of cases [8].

Before the widespread availability of CT, identification of small, incidental SFTP would have been rare, and thus this patient population is unlikely to have been included in historical studies.

The majority of papers were from surgical centres, which likely results in selection of patients in whom an intervention has already been deemed necessary. In this review, $98 \%$ of patients underwent surgical resection with paucity of information provided regarding either the decision to resect or timing of resection. As no comparative data are available, conclusions about pre-resection management or outcomes of conservatively managed patients cannot be derived. The two papers $[8,12]$ with malignancy rates of above $45 \%$ were from oncology referral centres, which may bias towards more aggressive disease and not represent the population of patients with SFTP as a whole. The rate of histological malignancy could therefore be lower than $30 \%$ in the general population.

\section{Future directions}

Modern immunohistochemistry has significantly improved diagnostic accuracy for SFTP. Identification of NAB2-STAT6 gene fusions may aid in refining diagnosis [37, 38], and the wealth of potential new tests, such as those using proliferation markers [34], could help to more reliably predict which patients will have a malignant clinical course.

Identification of radiological parameters associated with a benign clinical course might allow surgery to be avoided in some cases. ${ }^{18}$ PET-CT could be useful in predicting malignancy or targeting areas for biopsy [39].

The reported literature shows that it is common practice to resect SFTP, but focussing research on methods to help risk stratify patients may allow surgery to be avoided in some cases. Likewise, further investigation into the characteristics of patients who experience multiple episodes of recurrence could help to revise the treatment pathway for this subset.

\section{Recommendations}

In the authors' opinion, radiological follow-up should be undertaken using magnetic resonance imaging, where possible, to reduce the radiation dose. Scanning should occur at 3 months and 6 months (after diagnosis or resection), and then yearly thereafter for 10 years. Preoperative biopsies are not indicated to determine malignant potential of SFTP, due to the inconsistencies reported between biopsy and resection specimens but may be required to rule out an alternative diagnosis.

Further information is needed to risk stratify cases and therefore allow for individualised management, including the indication and timing of resection, the utility of oncological treatments and the optimal duration and schedule of follow-up.

\section{Conclusions}

This review demonstrates that there are large gaps in the evidence from the literature regarding management of SFTP. There are few robust predictors of a malignant clinical course, and preoperative biopsies often add little value. The data support long-term follow-up for patients after resection, but it may be necessary to extend follow-up to around 10 years. Further work on risk stratification will need to 
include radiologically diagnosed SFTP to establish whether any particular characteristic predicts a benign course, and therefore potentially avoid surgery. Cases where multiple recurrences occur despite adequate surgical intervention need to be studied more closely. This review shows a clear need for further studies to provide robust evidence to guide clinicians in the management of patients with SFTP.

Authors contributions: R.M. Mercer was responsible for initial draft preparation and revision. R.M. Mercer, C. Wigston and R. Banka were responsible for data acquisition. All authors were responsible for reviewing and approving the final manuscript. R.M. Mercer and N.M. Rahman are responsible for the overall content as guarantors.

Data availability: All data are already in the public domain.

Conflict of interest: R.M. Mercer has nothing to disclose. C. Wigston has nothing to disclose. R. Banka has nothing to disclose. G. Cardillo has nothing to disclose. R. Benamore has nothing to disclose. G. Cardillo has nothing to disclose. R. Asciak has nothing to disclose. M. Hassan has nothing to disclose. R.J. Hallifax has nothing to disclose. L. Wing has nothing to disclose. E.O. Bedawi has nothing to disclose. N.A. Maskell reports an unrestricted research grant from Rocket and Becton Dickinson for IPC plus HiSPEC and REDUCE studies, and sat on the advisory board for cook medical. E. Harriss has nothing to disclose. R.F. Miller reports personal fees for a nonpromotional lecture on clinical aspects of HIV infection from Gilead outside the submitted work. N.J. Rahman reports grants and personal fees from Rocket Medical UK, grants from BD USA, and personal fees from Lung therapeutics USA, outside the submitted work.

Support statement: No external funding was sought or required in relation to this analysis, its interpretation, and in the writing of this manuscript. R.J. Hallifax is funded by an NIHR Clinical Lectureship. R.F. Miller is supported by CNWL and Royal Free London NHS Foundation Trusts. N.M. Rahman is supported by the NIHR Oxford Biomedical Research Centre.

\section{References}

1 England DM, Hochholzer L, McCarthy MJ. Localized benign and malignant fibrous tumors of the pleura. A clinicopathologic review of 223 cases. Am J Surg Pathol 1989; 13: 640-658.

2 Bosman FTJE, Lakhani SR, Ohgaki H. WHO Classification of Tumours of the Lung, Pleura, Thymus and Heart. Lyons, France, International Agency for Research on Cancer (IARC), 2015.

3 Luciano C, Francesco A, Giovanni V, et al. CT signs, patterns and differential diagnosis of solitary fibrous tumors of the pleura. J Thorac Dis 2011; 2: 21-25.

4 Marchevsky AM, Khoor A, Walts AE, et al. Localized malignant mesothelioma, an unusual and poorly characterized neoplasm of serosal origin: best current evidence from the literature and the International Mesothelioma Panel. Mod Pathol 2020; 33: 281-296.

5 Yeom YK, Kim MY, Lee HJ, et al. Solitary fibrous tumors of the pleura of the thorax: CT and FDG PET characteristics in a tertiary referral center. Medicine (Baltimore) 2015; 94: e1548.

6 Cardillo G, Carbone L, Carleo F, et al. Solitary fibrous tumors of the pleura: an analysis of 110 patients treated in a single institution. Ann Thorac Surg 2009; 88: 1632-1637.

7 Boddaert G, Guiraudet P, Grand B, et al. Solitary fibrous tumors of the pleura: a poorly defined malignancy profile. Ann Thorac Surg 2015; 99: 1025-1031.

8 Bylicki O, Rouvière D, Cassier P, et al. Assessing the multimodal management of advanced solitary fibrous tumors of the pleura in a routine practice setting. J Thorac Oncol 2015; 10: 309-315.

9 Chu X, Zhang L, Xue Z, et al. Solitary fibrous tumor of the pleura: an analysis of forty patients. J Thorac Dis 2012; 4: $146-154$.

10 Demicco EG, Park MS, Araujo DM, et al. Solitary fibrous tumor: a clinicopathological study of 110 cases and proposed risk assessment model. Mod Pathol 2012; 25: 1298-1306.

11 Demicco EG, Wagner MJ, Maki RG, et al. Risk assessment in solitary fibrous tumors: validation and refinement of a risk stratification model. Mod Pathol 2017; 30: 1433-1442.

12 DeVito N, Henderson E, Han G, et al. Clinical characteristics and outcomes for solitary fibrous tumor (SFT): a single center experience. PLoS One 2015; 10: e0140362.

13 Enon S, Kilic D, Yuksel C, et al. Benign localized fibrous tumor of the pleura: report of 25 new cases. Thorac Cardiovasc Surg 2012; 60: 468-473.

14 Franzen D, Diebold M, Soltermann A, et al. Determinants of outcome of solitary fibrous tumors of the pleura: an observational cohort study. BMC Pulm Med 2014; 14: 138.

15 Gholami S, Cassidy MR, Kirane A, et al. Size and location are the most important risk factors for malignant behavior in resected solitary fibrous tumors. Ann Surg Oncol 2017; 13: 3865-3871.

16 Guo W, Xiao HL, Jiang YG, et al. Retrospective analysis for thirty-nine patients with solitary fibrous tumor of pleura and review of the literature. World J Surg Oncol 2011; 9: 134.

17 Gupta A, Souza CA, Sekhon HS, et al. Solitary fibrous tumour of pleura: CT differentiation of benign and malignant types. Clin Radiol 2017; 72: 796.e9-796e17.

18 Harrison-Phipps KM, Nichols FC, Schleck CD, et al. Solitary fibrous tumors of the pleura: results of surgical treatment and long-term prognosis. J Thorac Cardiovasc Surg 2009; 138: 19-25.

19 Helage S, Revel MP, Chabi ML, et al. Solitary fibrous tumor of the pleura: can computed tomography features help predict malignancy? A series of 56 patients with histopathological correlates. Diagn Interv Imaging 2016; 97: 347-353.

20 Kim J, Choi Y. Clinicopathological prognostic indicators of solitary fibrous tumor. Lab Invest 2017; 97: 18A.

21 Lahon B, Mercier O, Fadel E, et al. Solitary fibrous tumor of the pleura: outcomes of 157 complete resections in a single center. Ann Thorac Surg 2012; 94: 394-400.

22 Liu J, Cai C, Wang D, et al. Video-assisted thoracoscopic surgery (VATS) for patients with solitary fibrous tumors of the pleura. J Thorac Oncol 2010; 5: 240-243. 
23 McGuire A, Villeneuve PJ, Sekhon $\mathrm{H}$, et al. Predictors of malignant pathology and the role of trans-thoracic needle biopsy in management of solitary fibrous tumors of the pleura: a 30-year review of a tertiary care center patient cohort. Open J Thorac Surg 2016; 06: 13.

24 O'Neill AC, Tirumani SH, Do WS, et al. Metastatic patterns of solitary fibrous tumors: a single-institution experience. AJR Am J Roentgenol 2017; 208: 2-9.

25 Rena O, Filosso PL, Papalia E, et al. Solitary fibrous tumour of the pleura: surgical treatment. Eur J Cardiothorac Surg 2001; 19: 185-189.

26 Santos RS, Haddad R, Lima CE, et al. Patterns of recurrence and long-term survival after curative resection of localized fibrous tumors of the pleura. Clin Lung Cancer 2005; 7: 197-201.

27 Schmid S, Csanadi A, Kaifi JT, et al. Prognostic factors in solitary fibrous tumors of the pleura. J Surg Res 2015, 195: 580-587.

28 Tan F, Wang Y, Gao S, et al. Solitary fibrous tumors of the pleura: a single center experience at National Cancer Center, China. Thorac Cancer 2018; 9: 1763-1769.

29 Tapias LF, Mino-Kenudson M, Lee H, et al. Risk factor analysis for the recurrence of resected solitary fibrous tumours of the pleura: a 33-year experience and proposal for a scoring system. Eur J Cardiothorac Surg 2013; 44: 111-117.

30 Vejvodova S, Spidlen V, Mukensnabl P, et al. Solitary fibrous tumor - less common neoplasms of the pleural cavity. Ann Thorac Cardiovasc Surg 2017; 23: 12-18.

31 Scarsbrook AF, Evans AL, Slade M, et al. Recurrent solitary fibrous tumour of the pleura due to tumour seeding following ultrasound-guided transthoracic biopsy. Clin Radiol 2005; 60: 130-132.

32 Lococo F, Cardillo G, Spaggiari L, et al. Malignant solitary fibrous tumors: clinical characteristics, surgical treatment and long-term outcome in a multi-centric series of 50 patients. Eur Surg Res 2012; 49: 186.

33 de Perrot M, Fischer S, Brundler MA, et al. Solitary fibrous tumors of the pleura. Ann Thorac Surg 2002; 74 285-293.

34 Diebold M, Soltermann A, Hottinger S, et al. Prognostic value of MIB-1 proliferation index in solitary fibrous tumors of the pleura implemented in a new score - a multicenter study. Respir Res 2017; 18: 210.

35 Bellini A, Marulli G, Breda C, et al. Predictors of behaviour in solitary fibrous tumours of the pleura surgically resected: analysis of 107 patients. J Surg Oncol 2019; 120: 761-767.

36 Reisenauer JS, Mneimneh W, Jenkins S, et al. Comparison of risk stratification models to predict recurrence and survival in pleuropulmonary solitary fibrous tumor. J Thorac Oncol 2018; 13: 1349-1362.

37 Rekhi B, Shetty O, Tripathi P, et al. Molecular characterization of a series of solitary fibrous tumors, including immunohistochemical expression of STAT6 and NATB2-STAT6 fusion transcripts, using Reverse Transcriptase (RT)-Polymerase chain reaction(PCR) technique: an Indian experience. Pathol Res Pract 2017; 213: 1404-1411.

38 Tai HC, Chuang IC, Chen TC, et al. NAB2-STAT6 fusion types account for clinicopathological variations in solitary fibrous tumors. Mod Pathol 2015; 28: 1324-1335.

39 Fei B, Schuster DM. PET molecular imaging-directed biopsy: a review. AJR Am J Roentgenol 2017; 209: 255-269. 